\title{
A REST-based Framework to Support Non-Invasive and Early Coeliac Disease Diagnosis
}

\author{
Salvatore Sorce \\ Università degli Studi di Palermo \\ Palermo, Italy \\ salvatore.sorce@unipa.it \\ Angele Giuliano \\ AcrossLimits \\ Naxxar, Malta \\ angele@acrosslimits.com \\ Domenico Tegolo \\ Università degli Studi di Palermo \\ Palermo, Italy \\ domenico.tegolo@unipa.it
}

\author{
Vito Gentile \\ Università degli Studi di Palermo \\ Palermo, Italy \\ vito.gentile@unipa.it \\ Marco Elio Tabacchi \\ Università degli Studi di Palermo \\ Palermo, Italy \\ marcoelio.tabacchi@unipa.it \\ Cesare Valenti \\ Università degli Studi di Palermo \\ Palermo, Italy \\ cesare.valenti@unipa.it
}

\author{
Donato Cascio \\ Università degli Studi di Palermo \\ Palermo, Italy \\ donato.cascio@unipa.it \\ Vincenzo Taormina \\ Università degli Studi di Palermo \\ Palermo, Italy \\ vincenzo.taormina@unipa.it \\ Giuseppe Raso \\ Università degli Studi di Palermo \\ Palermo, Italy \\ giuseppe.raso@unipa.it
}

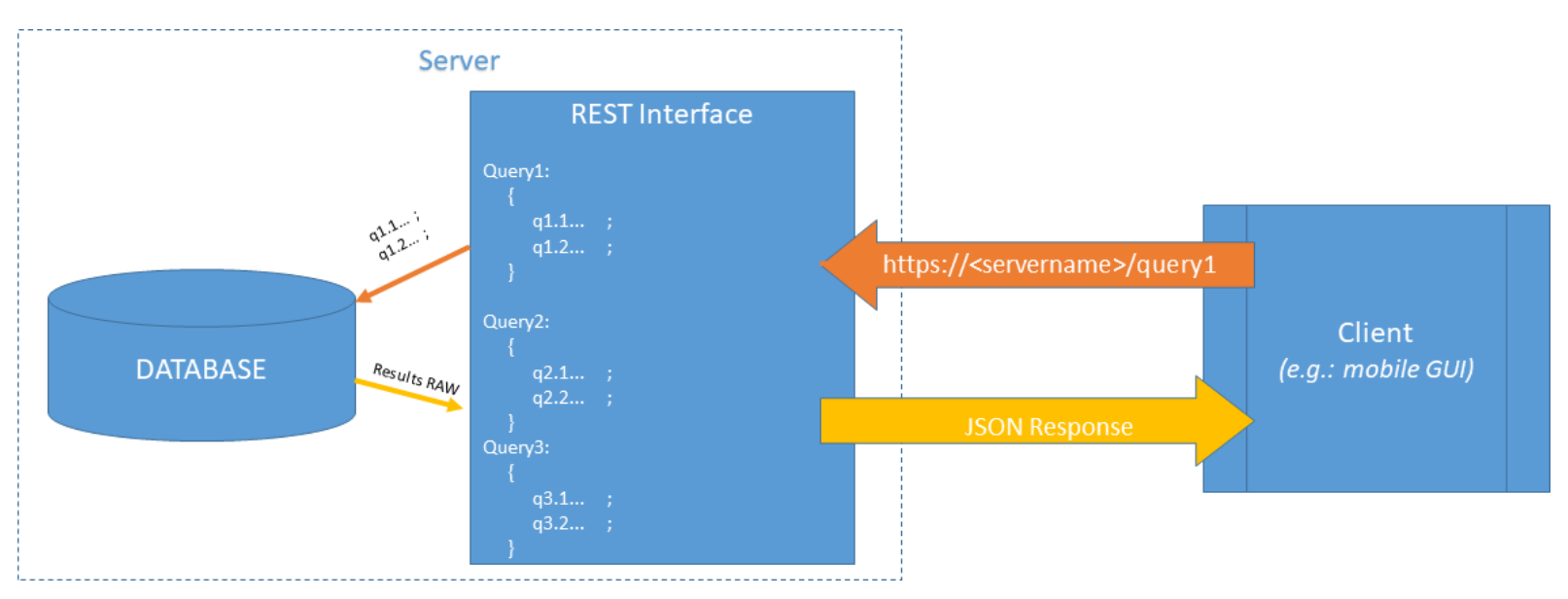

Figure 1: Architecture of our framework.

\begin{abstract}
The health sector has traditionally been one of the early adopters of databases, from the most simple Electronic Health Record (formerly Computer-Based Patient Record) systems in use in general practice, hospitals and intensive care units to big data, multidata based systems used to support diagnosis and care decisions. In this paper we present a framework to support non-invasive and early diagnosis of coeliac disease. The proposed framework makes use of
\end{abstract}

Permission to make digital or hard copies of all or part of this work for personal or classroom use is granted without fee provided that copies are not made or distributed for profit or commercial advantage and that copies bear this notice and the full citation on the first page. Copyrights for components of this work owned by others than the author(s) must be honored. Abstracting with credit is permitted. To copy otherwise, or republish, to post on servers or to redistribute to lists, requires prior specific permission and/or a fee. Request permissions from permissions@acm.org.

CompSysTech '19, June 21-22, 2019, Ruse, Bulgaria

(c) 2019 Copyright held by the owner/author(s). Publication rights licensed to ACM. ACM ISBN 978-1-4503-7149-0/19/06 ..\$15.00

https://doi.org/10.1145/3345252.3345296 well-known technologies and techniques, both hardware and software, put together in a novel way. The main goals of our framework are: (1) providing users with a reliable and fast repository of a large amount of data; (2) to make such repository accessible by means of a suitable API in multiple modes, such as intuitive web-based or mobile visual interfaces; (3) to allow for data processing and analysis, as a basis for decision support systems.

\section{CCS CONCEPTS}

- Information systems $\rightarrow$ Database design and models; Application servers; Information systems applications; Storage architectures; $\bullet$ Applied computing $\rightarrow$ Health care information systems.

\section{KEYWORDS}

Health care systems, Databases, coeliac disease diagnosis support 
ACM Reference Format:

Salvatore Sorce, Vito Gentile, Donato Cascio, Angele Giuliano, Marco Elio Tabacchi, Vincenzo Taormina, Domenico Tegolo, Cesare Valenti, and Giuseppe Raso. 2019. A REST-based Framework to Support Non-Invasive and Early Coeliac Disease Diagnosis. In Proceedings of the 20th International Conference on Computer Systems and Technologies (CompSysTech '19), June 21-22, 2019, Ruse, Bulgaria. ACM, New York, NY, USA, 6 pages. https: //doi.org/10.1145/3345252.3345296

\section{INTRODUCTION}

During last years, autoimmune diseases are becoming more and more common all over the world. In particular, in the Mediterranean area they have a high prevalence probably due to the marked presence of gluten in the Mediterranean diet [28]. Among autoimmune diseases, the coeliac disease stands out with an estimated prevalence of about $1 \%$ [10], symptoms within the first five years of life in $50 \%$ of cases [25] and a higher incidence among women $(60 \%$ more with respect to men). Extra-gastrointestinal symptoms and autoimmune complications related to the delayed diagnosis result in low quality of life and high healthcare costs. As a consequence, there is a need for early detection of coeliac disease in order to both improve the quality of life and to reduce the costs.

On the other hand, a reliable diagnosis of coeliac disease is currently carried out by means of the recognition of symptoms, serological tests and final confirmation with histological examination of intestinal biopsies. The ESPGHAN ${ }^{1}$ guidelines [16] report the possibility of avoiding the biopsy for the diagnosis of coeliac disease in children that are genetically predisposed and with high titers of anti-tTG IgA antibodies, after confirmation of the positivity with another test performed by a different method (anti-endomysial antibodies, EMA). Unfortunately, such a test is observer-dependent and furthermore it is possible to apply this protocol only on a limited number of patients with high probability of coeliac disease deduced by their symptoms.

As a consequence, there is a need for:

- an early and reliable diagnosis of coeliac disease;

- avoiding invasive tests, such as biopsy;

- lowering treatment costs due to a late diagnosis.

There are several obstacles to fulfilling these needs. First, the accuracy of a test, in terms of sensitivity and specificity, does not allow for the estimation of its predictive reliability without taking into account the symptoms. Next, currently there is a lack of statistical (predictive) information to calculate the a priori probability of the disease, of its symptoms, or both of them. Furthermore, the increasing complexity and size of needed data require new computing solutions from information technology and computer science to support physicians during the diagnostic process. Last, despite in some cases (less than 10\%) the intestinal biopsy could be avoided under certain conditions, it still remains the Gold Standard for the diagnosis of coeliac disease.

In this paper we present a framework aiming at supporting the non-invasive and early diagnosis of the coeliac disease. Our framework is part of a more general project (see Acknowledgments) being developed in the Mediterranean area, which involves more

\footnotetext{
${ }^{1}$ European Society for Paediatric Gastroenterology Hepatology and Nutrition
}

than twenty thousand school-age children. The main goals of the project are:

- increasing the number of people correctly diagnosed with coeliac disease by means of a PoCT (Point-of-Care Test), thus improving their quality of life and reducing the treatment costs

- reducing the use of biopsy for the coeliac disease diagnosis, thus reducing the diagnosis invasivity and costs

- improving the diagnostic accuracy and reliability of the PoCT, even if it results negative

In particular, our framework will provide for a database as a foundation for the collection of a large amount of data and for any kind of subsequent data analysis and processing, such as a Decision Support System (DSS). As a side goal, the introduction and sharing of ICT tools and services and in particular a database storing a large amount of data from a survey on a significant sample could help the scientific community in finding new evidences that can lead to further improvements of the diagnostic process of the coeliac disease.

\section{RELATED WORK}

\section{1 e-Health and databases}

The health sector has traditionally been one of the early adopters of databases, from the most simple Electronic Health Record (formerly Computer-Based Patient Record) systems in use in general practice, hospitals and intensive care units up to big data, multidata based systems used to support diagnosis and care decisions. The term $e$ health has been coined in order to acknowledge such a development. A discussion of historical relevance can be found in [5], a discussion on relative ethic concerns in [17] while a more recent contribution is [23].

A number of Medical Databases (MDBs) are already implemented in the field of general practice in different countries: notable examples are UK's Clinical Practice Research Datalink (CPRD [15], formerly the General Practice Research Database), that covers more than 600 practices with data ranging from 1987 to the present, and QResearch ${ }^{2}$. Such MDBs have been extensively used in cross analysis. Recent examples include the correlation between the use of oral contraceptives and the risk of venous thromboembolism [29], the risk of contracting hepatocellular carcinoma in cirrhosis patients [30]. A MDB containing real ultrasound imaging was created to study the correlations between chromosomal defects such as trisomy 13, 18 and 21 with respect to anomalies in the nuchal translucency thickness [20]. An analogous work on a videocapillaroscopic database allowed to highlight that alterations in the peripheral microcirculation are closely related to lichen planus, pemphigus and pemphigoid, diabetes, hypercholesterolemia, scleroderma, Sjögren's syndrome and rheumatoid arthritis [3].

The multiplication of e-health systems has accelerated the need for a way of exchanging data among systems with ease. A number of solutions have been put forward; among them, Fast Healthcare Interoperability Resources (FHIR, now at version $3^{3}$ ) is a draft standard that has recently gained attention, and is of interest in

\footnotetext{
${ }^{2} \mathrm{http}: / /$ www.qresearch.org (last retrieved: May 18th, 2019)

${ }^{3}$ http://hl7.org/fhir/ (last retrieved: May 18th, 2019)
} 
this context as it offers an API for exchanging health record that includes an HTTP-based RESTful protocol.

\subsection{ICT Solutions for coeliac Disease Diagnosis}

In recent years the research for a smarter, less invasive diagnosis of autoimmune diseases, and in particular of the coeliac disease, has been directed towards the use of ICT solutions generally based on classification and other statistical learning means [6], as well as the use of Decision Support Systems to facilitate the diagnosis. A number of such solutions are listed hereafter, without pretension of completeness. For a deeper discussion, a current survey on computer-aided decision support for the diagnosis of the coeliac disease based on endoscopic images can be found in [14]. The same research group [12] have previously investigated the impact of endoscope distortion correction on computer-assisted coeliac disease diagnosis. In [19] a more general discussion on computer-aided Decision Support Systems for endoscopy in the gastrointestinal tract also discuss the coeliac disease. Hegenbart et al. [13] have also analysed the impact of duodenal image capturing techniques and duodenal regions on the performance of automated diagnosis of the coeliac disease, devising an optimal combination of image capturing technique, duodenal region and feature extraction methods for automated coeliac disease diagnosis.

Vecsei, Fuhrmann and Uhl [27] have developed various techniques for texture classification which can be used for automated classification of endoscopic images for diagnosis of coeliac disease. The research group have worked also using feature extraction techniques based on the selection of highly discriminant Fourier filters in duodenal imagery, with the application of a range of different classifiers in a multiclassification approach in order to better diagnose coeliac disease [26], as well as on an approach based on convolutional neural networks [31].

In [1], the authors have used large datasets assaying single nucleotide polymorphisms (SNPs), to developed, using a statistical learning approach, genomic risk scores based on multiple SNPs that can more accurately predict coeliac disease risk across several populations in "real world" clinical settings.

Tenorio et al. [24] developed a clinical decision support system integrated with an automated classifier to recognize coeliac disease cases by selecting from five different experimental models developed using intelligence artificial techniques and selecting as the most accurate method the averaged one-dependence estimator (AODE) algorithm.

\section{THE PROPOSED FRAMEWORK}

The framework presented in this paper consists of two basic components:

- a relational database, for data storage;

- a web server, that implements the REST (Representational State Transfer) interface.

Both such components can be viewed as a unique entity, which serves data to many different clients. Indeed, one of the key requirements for our framework is to serve a wide and heterogeneous range of devices, applications and users. Each client (who can be a human user or an automatic data processing application) will then be able to add new information or read historical data, possibly analyzing and/or refining them. Of course, all these operations need to follow a well-defined set of privileges, which can be also defined in the database, along with information for user authentication. This architecture is depicted in Figure 1.

In this section we provide the design and implementation details of such a framework, including also some use cases to explain how the framework can be used by any web-based application.

\subsection{The Database}

The current schema of the database is composed of nine tables. The details of such tables are reported in Figure 2. In order to make the database as much interoperable and maintainable as possible, we used some well-known best practices:

- all table names start with a lowercase 't';

- all field names do not contain non-ASCII characters, and they are composed (as much as possible) of meaningful groups of characters, making it easier to understand the data they represent and the table they belong to.

The schema takes into account all known constraints and requirements both from ethical, technical and functional points of view, and at the same time is ready to be extended for further functions to be supported according to the project's goals. In particular, the current version allows for collecting data and keep them suitably separated for security and privacy purposes, also allowing for different details collection among the different project's partners, if needed. Based on this schema, it is possible to build all the queries needed to achieve the project's goals, in terms of suitably structured data storage, and information extraction for statistical analysis and support to the early diagnosis process.

The database is deployed over a MariaDB server environment. We decided to use this solution because it is fully open source, thus allowing us to achieve the project's goal of continued free and open maintainability, with the same operational and interoperability features of MySQL [2].

\subsection{RESTful API}

As mentioned, one of the main requirements for our framework is to be usable from a wide and heterogeneous range of applications. To this end, we decided to implement a set of services based on the REST paradigm [7]. This paradigm allows for the easy building of web-based solutions in different application fields, such as public displays [8], cultural heritage [9], service access [22], and many others [21]. With this model, a client can be implemented using any programming language, as long as it allows to send HTTP requests and process HTTP responses.

Driven by this principle, we implemented a web server using the Python programming language and its module Flask [11]. With this tools we defined a set of REST API, summarized in Table 1.

Each URL was mapped to a specific HTTP method. In particular, we exploited the natural mapping between the CRUD operations (Create, Read, Update, Delete) and the HTTP methods, respectively POST, GET, PUT, DELETE. Some requests have also a variable number of parameters, each of which needs to be properly included in the request body. All the HTTP responses were structured using the JSON markup language [4], using the following template: 


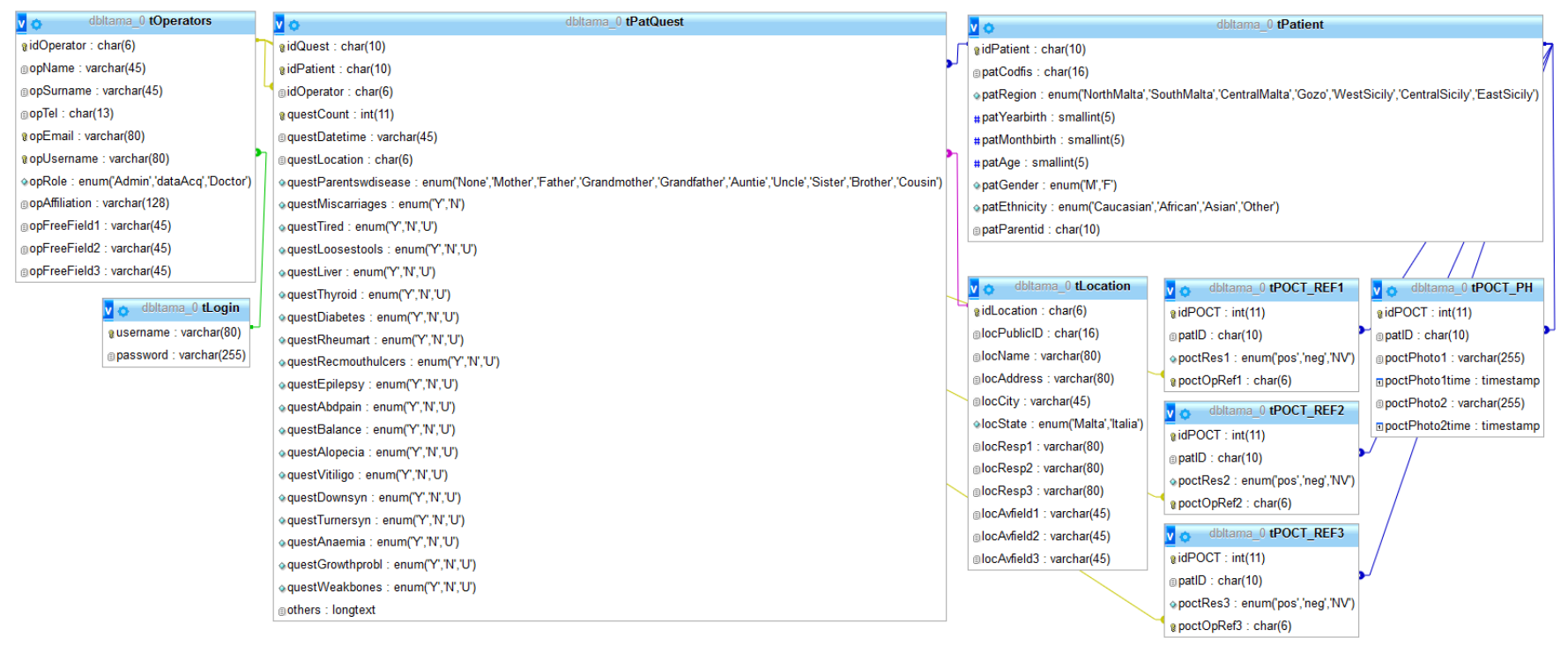

Figure 2: Database ER schema.

\{

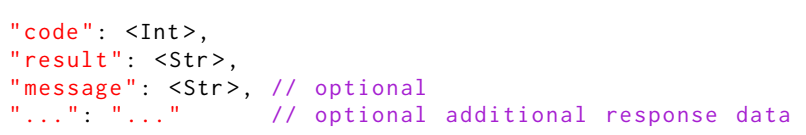

\subsection{REST-based access}

In this section we exemplify a use case where a multi-user application apply our framework to add and retrieve information about patients.

3.3.1 Example application. Our example application may consist in a set of views, each of which can be seen as a form, where the user can input data, or read results of her requests. The views of our application are the following ones:
Login This view includes a form where the user can input an already registered username, or decide to register a new one. In both cases, she has to provide also a password

Add Patient This view allows to add demographic information about a new patient

Questionnaire This view allows a patient to answer to a questionnaire, in order to collect information about her historical health data

PoCT This view allows a nurse or a doctor to add information about the results of a point of care test, including also pictures of such test (see Figure 3)

Analysis This view allows a doctor to analyze patient data and to adjust some of the information if needed

3.3.2 Use case. Through the login view, a user can add her username and password, which are used as parameters for the Login service described in Table 1. This implies, of course, that the user must be already registered in the database. If this is not the case, a

Table 1: REST services implemented in our framework.

\begin{tabular}{|l|l|c|l|}
\hline Service Name & URL & HTTP Method & Description \\
\hline Registration & /auth/register & POST & Allows to register a new username with password \\
\hline Login & /auth/login & POST & $\begin{array}{l}\text { Allow to login with username and password, and returns a token to } \\
\text { be used for authentication in following requests }\end{array}$ \\
\hline Get role & /role & GET & $\begin{array}{l}\text { Returns the role of a user. Useful for gathering information about } \\
\text { user's permissions }\end{array}$ \\
\hline Update role & /role & PUT & Update the role of a user \\
\hline Add new patient & /patient & POST & Allows to add information about a patient \\
\hline Get patient data & /patient & GET & Allows to retrieve information about a patient \\
\hline Update patient information & /patient & PUT & Allows to update patient information \\
\hline Add new questionnaire & /quest & POST & Allows to add patient information collected via a questionnaire \\
\hline Add new PoCT & /poct & POST & $\begin{array}{l}\text { Allows to add patient information collected via a Point of Care Test } \\
\text { (PoCT) }\end{array}$ \\
\hline
\end{tabular}



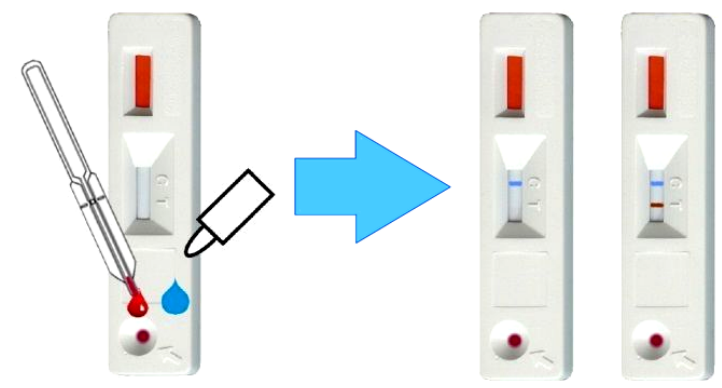

Figure 3: Usage of Point of Care Test (PoCT). A blood sample is added to the PoCT and after few minutes one or two lines appear. Pictures of the PocT results can be also be added to the database using an HTTP POST request.

registration needs to be done by means of the Registration service. It is worth noting that both the Login and Registration services need a mechanism for securing the transmission of users' credentials. This can be done in a plethora of ways, but in our case we opted for the SSL encryption provided by the HTTPS protocol.

Once the Login request is sent, if the credentials are correct the JSON response will contain a code attribute set to 0 , and another attribute named token, which is then used as a parameters of all the subsequent requests, in order to identify the user. This token-based authentication is inspired by the JSON Web Token standard [18].

After the login, the application sends a HTTP GET request to the Get role service, in order to obtain the permissions associated with the user to add or retrieve specific data. This role is used by the application in order to filter some of the available resources and views. For instance, a user with a role of "nurse" can access the PoCT view, but cannot access the Analysis view (which is only accessible for users with the role of "doctor").

Assuming that this application is used during a screening session for assessing coeliac disease, a user can add patient information through the Add Patient service. Then, if for instance the application is run inside a tablet, a questionnaire aimed at collecting information about patient's medical history can be submitted by the patient herself. Finally, the PoCT service allows doctor or nurse to submit the information about the Point of Care test.

In a second phase, possibly after the data collection, one or more doctors can start analyzing the data, using the Analysis view. Here, patients' data can be displayed, read and (if needed) also corrected by the doctor.

\section{CONCLUSION AND FUTURE WORK}

This contribution intends to present an architecture oriented to the fusion of heterogeneous data involved in the Smart Healthcare domain. It contains a secure protocol on a high-performance public network, a data center for data storage, and allows for straightforward data collection and information extraction by means of peripheral devices. This approach made it possible to divide the tasks into three macro areas: data acquisition (via devices with a versatile interface), a communication unit between the acquisition devices and the remote system in which the data can be stored and a functional unit for data storage. The aggregation of these components has allowed designing and implementing a framework capable of satisfying the needs of users with different skills through specific interfaces. The need to collect data from heterogeneous sources (images, paper, digital, ...) made it necessary to define a robust, structured (yet versatile) and hierarchical architecture, in order to be able to merge such data from various sites into a single repository. In such a framework all heterogeneous data of a patient will be aggregated in local mode and subsequently uploaded to the data center through the network connection module. Moreover, to make the framework be a valid support for the doctor's decisionmaking phase, a functional module called DSS will be included as a module of data center activities (see Figure 4). The DSS will be characterized by inferential rules that can suggest to the doctor the right path to follow in order to diagnose the pathology with a high degree of correctness and at the same time not subjecting the child to biopsy. Currently, the preliminary version of the entire system and the framework are being tested, but future updates are planned on the process management and on the DSS. Specifically, the rules of the DSS will be designed to ensure its further use in medical settings where invasive examinations are currently present both in adolescent and non-adolescent age.

\section{ACKNOWLEDGMENTS}

This paper has been partially supported by the ITAMA project under the international cooperation program INTERREG V-A (JSCode C1-1.1-18). The content of this publication does not reflect the official opinion of the European Union. Responsibility for the information and views expressed lies entirely with the author(s).

\section{REFERENCES}

[1] Gad Abraham, Jason A Tye-Din, Oneil G Bhalala, Adam Kowalczyk, Justin Zobel, and Michael Inouye. 2014. Accurate and robust genomic prediction of celiac disease using statistical learning. PLoS genetics 10, 2 (2014), e1004137.

[2] Daniel Bartholomew. 2012. MariaDB VS. MySQL. Technical Report.

[3] Fabio Bellavia, Cacioppo Antonino, Carmen Alina Lupascu, Pietro Messina, Giuseppe Alessandro Scardina, Domenico Tegolo, and Cesare Valenti. 2014. A non-parametric segmentation methodology for oral videocapillaroscopic images. Computer Methods and Programs in Biomedicine 114, 3 (2014), 240-246.

[4] Douglas Crockford. 2006. The application/json Media Type for JavaScript Object Notation (fSON). RFC 4627. https://doi.org/10.17487/RFC4627

[5] Molla S Donaldson, Kathleen N Lohr, et al. 1994. Health data in the information age: use, disclosure, and privacy. National Academies.

[6] Amel Benammar Elgaaied, Donato Cascio, Salvatore Bruno, Maria Cristina Ciaccio, Marco Cipolla, Alessandro Fauci, Rossella Morgante, Vincenzo Taormina, Yousr Gorgi, Raja Marrakchi Triki, Melika Ben Ahmed, Hechmi Louzir, Sadok Yalaoui, Sfar Imene, Yassine Issaoui, Ahmed Abidi, Myriam Ammar, Walid Bedhiafi, Oussama Ben Fraj, Rym Bouhaha, Khouloud Hamdi, Koudhi Soumaya, Bilel Neili, Gati Asma, Mariano Lucchese, Maria Catanzaro, Vincenza Barbara, Ignazio Brusca, Maria Fregapane, Gaetano Amato, Giuseppe Friscia, Trai Neila, Souayeh Turkia, Haouami Youssra, Raja Rekik, Hayet Bouokez, Maria Vasile Simone, Francesco Fauci, and Giuseppe Raso. 2016. Computer-Assisted Classification Patterns in Autoimmune Diagnostics: The AIDA Project. BioMed Research International 2016 (2016), 1-9. https://doi.org/10.1155/2016/2073076

[7] Xinyang Feng, Jianjing Shen, and Ying Fan. 2009. REST: An alternative to RPC for Web services architecture. In 2009 First International Conference on Future Information Networks. 7-10. https://doi.org/10.1109/ICFIN.2009.5339611

[8] Vito Gentile, Mohamed Khamis, Salvatore Sorce, and Florian Alt. 2017. They are looking at me!. In Proceedings of the 6th ACM International Symposium on Pervasive Displays - PerDis '17. ACM Press. https://doi.org/10.1145/3078810. 3078822

[9] Vito Gentile, Salvatore Sorce, Giuseppe Russo, Dario Pirrone, and Antonio Gentile. 2016. A Multimodal Fruition Model for Graphical Contents in Ancient Books. In Proceedings of the 17th International Conference on Computer Systems and Technologies 2016 - CompSysTech '16. ACM Press. https://doi.org/10.1145/2983468. 2983477 


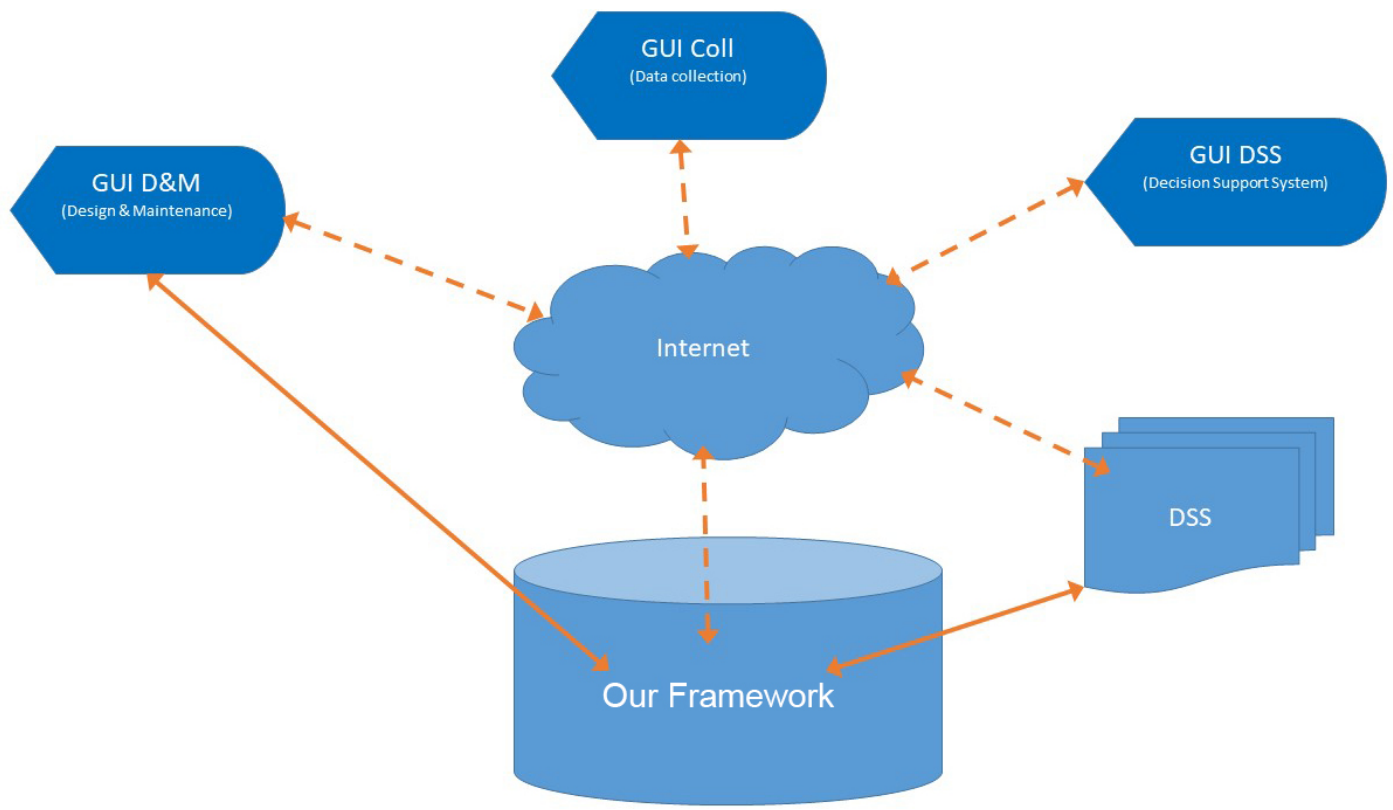

Figure 4: Projected contextualization of our framework.

[10] Luigi Greco, Laura Timpone, Abdelhak Abkari, Mona Abu-Zekry, Thomas Attard Faouzi Bouguerrà, Paskal Cullufi, Aydan Kansu, Dusanka Micetic-Turk, Zrinjka MiĄąak, Eleftheria Roma, Raanan Shamir, and Selma Terzic. 2011. Burden of celiac disease in the Mediterranean area. World fournal of Gastroenterology 17 45 (2011), 4971. https://doi.org/10.3748/wjg.v17.i45.4971

[11] Miguel Grinberg. 2018. Flask Web Development: Developing Web Applications with Python. " O'Reilly Media, Inc.".

[12] Michael Gschwandtner, Michael Liedlgruber, Andreas Uhl, and Andreas Vécsei. 2010. Experimental study on the impact of endoscope distortion correction on computer-assisted celiac disease diagnosis. In Proceedings of the 10th IEEE International Conference on Information Technology and Applications in Biomedicine. IEEE, 1-6.

[13] S Hegenbart, R Kwitt, M Liedlgruber, A Uhl, and A Vécsei. 2009. Impact of duodenal image capturing techniques and duodenal regions on the performance of automated diagnosis of celiac disease. In 2009 Proceedings of 6th International Symposium on Image and Signal Processing and Analysis. IEEE, 718-723.

[14] Sebastian Hegenbart, Andreas Uhl, and Andreas Vécsei. 2015. Survey on computer aided decision support for diagnosis of celiac disease. Computers in biology and medicine 65 (2015), 348-358.

[15] Emily Herrett, Arlene M Gallagher, Krishnan Bhaskaran, Harriet Forbes, Rohin Mathur, Tjeerd van Staa, and Liam Smeeth. 2015. Data resource profile: clinical practice research datalink (CPRD). International journal of epidemiology 44, 3 (2015), 827-836.

[16] S. Husby, S. Koletzko, I.R. Korponay-Szabó, M.L. Mearin, A. Phillips, R. Shamir, R. Troncone, K. Giersiepen, D. Branski, C. Catassi, M. Lelgeman, M. Mäki, C. RibesKoninckx, A. Ventura, and K.P. Zimmer. 2012. European Society for Pediatric Gastroenterology, Hepatology, and Nutrition Guidelines for the Diagnosis of Coeliac Disease. Fournal of Pediatric Gastroenterology and Nutrition 54, 1 (jan 2012), 136-160. https://doi.org/10.1097/mpg.0b013e31821a23d0

[17] Julia Inthorn, Rudolf Seising, and Marco Elio Tabacchi. 2015. Having the final say: Machine support of ethical decisions of doctors. In Machine Medical Ethics, Simon van Rysewyk and Mattjis Pontier (Eds.). Intelligent Systems, Control and Automation: Science and Engineering, Vol. 74. Springer, 181-206. https //doi.org/10.1007/978-3-319-08108-3

[18] M. Jones, J. Bradley, and N. Sakimura. 2015. JSON Web Token (JWT). RFC 7519 https://doi.org/10.17487/RFC7519

[19] Michael Liedlgruber and Andreas Uhl. 2011. Computer-aided decision support systems for endoscopy in the gastrointestinal tract: a review. IEEE reviews in biomedical engineering 4 (2011), 73-88.

[20] Giuseppa Sciortino, Domenico Tegolo, and Cesare Valenti. 2017. Automatic detection and measurement of nuchal translucency. Computers in Biology and Medicine 82 (2017), 12-20.
[21] Rosario Sorbello, Salvatore Tramonte, Marcello Emanuele Giardina, Vincenzo La Bella, Rossella Spataro, Brendan Allison, Christoph Guger, and Antonio Chella. 2018. A Human-Humanoid Interaction Through the Use of BCI for LockedIn ALS Patients Using Neuro-Biological Feedback Fusion. IEEE Transactions on Neural Systems and Rehabilitation Engineering 26, 2 (Feb. 2018), 487-497. https://doi.org/10.1109/tnsre.2017.2728140

[22] Salvatore Sorce, Agnese Augello, Antonella Santangelo, Giovanni Pilato, Antonio Gentile, Alessandro Genco, and Salvatore Gaglio. 2007. A multimodal guide for the augmented campus. In Proceedings of the 35th annual ACM SIGUCCS conference on User services - SIGUCCS '07. ACM Press. https://doi.org/10.1145/1294046.1294123

[23] Joseph Tan. 2010. Developments in Healthcare Information Systems and Technologies: Models and Methods: Models and Methods. IGI Global.

[24] Josceli Maria Tenório, Anderson Diniz Hummel, Frederico Molina Cohrs, Vera Lucia Sdepanian, Ivan Torres Pisa, and Heimar de Fátima Marin. 2011. Artificial intelligence techniques applied to the development of a decision-support system for diagnosing celiac disease. International journal of medical informatics 80, 11 (2011), 793-802.

[25] Francesca Tucci, Luca Astarita, Abdelhak Abkari, Mona Abu-Zekry, Thomas Attard, Mongi Ben Hariz, José Ramon Bilbao, Ghazalia Boudraa, Samir Boukthir, Stefano Costa, Veselinka Djurisic, Jean-Pierre Hugot, Iñaki Irastorza, Aydan Kansu, Sanja Kolaček, Giuseppe Magazzù, Dušanka Mičetić-Turk, Zrinjka Misak, Eleftheria Roma, Pasqualino Rossi, Selma Terzic, Virtut Velmishi, Carmela Arcidiaco, Renata Auricchio, and Luigi Greco. 2014. Celiac disease in the Mediterranean area. BMC Gastroenterology 14, 1 (feb 2014). https://doi.org/10.1186/1471-230x-14-24

[26] Andreas Vécsei, Thomas Fuhrmann, Michael Liedlgruber, Leonhard Brunauer, Hannes Payer, and Andreas Uhl. 2009. Automated classification of duodenal imagery in celiac disease using evolved fourier feature vectors. Computer methods and programs in biomedicine 95, 2 (2009), S68-S78.

[27] A Vécsei, T Fuhrmann, and A Uhl. 2008. Towards automated diagnosis of celiac disease by computer-assisted classification of duodenal imagery. (2008).

[28] Alessandro Ventura, Giuseppe Magazzù, and Luigi Greco. 1999. Duration of exposure to gluten and risk for autoimmune disorders in patients with celiac disease. Gastroenterology 117, 2 (aug 1999), 297-303. https://doi.org/10.1053/ gast.1999.0029900297

[29] Yana Vinogradova, Carol Coupland, and Julia Hippisley-Cox. 2015. Use of combined oral contraceptives and risk of venous thromboembolism: nested casecontrol studies using the QResearch and CPRD databases. bmj 350 (2015), h2135.

[30] Joe West, Timothy R Card, Guruprasad P Aithal, and Kate M Fleming. 2017. Risk of hepatocellular carcinoma among individuals with different aetiologies of cirrhosis: a population-based cohort study. Alimentary pharmacology \& therapeutics 45, 7 (2017), 983-990.

[31] Georg Wimmer, Andreas Vécsei, and Andreas Uhl. 2016. CNN transfer learning for the automated diagnosis of celiac disease. In 2016 Sixth International Conference on Image Processing Theory, Tools and Applications (IPTA). IEEE, 1-6. 